\title{
The Wind Characteristics of Tall Cylinder Buildings with Vertical-Grooves
}

\author{
Weibin YUAN \\ College of Architecture and Civil Engineering \\ Zhejiang University of Technology \\ Hangzhou, PR China \\ Key Laboratory of Civil Engineering Structures \& \\ Disaster Prevention and Mitigation \\ E-mail: yuanwb@zjut.edu.cn
}

\author{
Hao CHEN \\ College of Architecture and Civil Engineering \\ Zhejiang University of Technology \\ Hangzhou, PR China
}

\author{
Zhao WANG \\ College of Architecture and Civil Engineering \\ Zhejiang University of Technology \\ Hangzhou, PR China
}

\begin{abstract}
The Reynolds number of flow field around circular cylinders is usually high under strong wind for most full-scale tall buildings, which is difficult to achieve in most wind tunnel tests. To explore the wind characteristics of tall cylindrical buildings with vertical-equidirectional grooves from subcritical to transcritical flow, wind tunnel tests and full-scale large eddy simulations were carried out. The results showed that the rectangular-grooves narrow the wake width due to the downstream movement of the separation point and the deeper grooves cause smaller mean pressure. Furthermore, larger groove roughness leads to lower frequency of vortex shedding and the Strouhal number remains at the range from 0.15 to 0.35.The drag coefficient of rough cylinders is $2 \sim 3$ times as large as that of smooth cylinders under the trend of decrease.
\end{abstract}

Keywords-tall cylinder buildings; wind characteristics; wind tunnel test; large eddy simulation

\section{INTRODUCTION}

For nearly half a century, many high rise buildings have been constructed, which have the characteristics of complicated facades and vertical balconies. As a result, the wind characteristics of the buildings may be influenced integrally and locally by surface roughness. Particular attention was paid to wind load around a tall circular building owing to its unique characteristics, which has been the subject of intense research, mostly by using experiments and numerical simulation.

Previously, some experiments have been devoted to the analysis of flow around a cylinder. Reference [1] carried out an experimental study to examine the reduction of wind pressures on glass and claddings of buildings with rectangular cross section with various roughness types. Reference [2] used cylinders with various equidirectional surface roughness and sizes of grooves to investigate the Strouhal number in the wake flow of a cylinder over a wide range of Reynolds numbers. Reference [3] did a similar work but focused on the vortex shedding from smooth and rough cylinders.

Apart from experimental studies, Computational Fluid Dynamics (CFD) has been extensively used as an efficient tool for the prediction of fluid characteristics in wind engineering [4-7]. Among available turbulence models, the theory of Large Eddy Simulation (LES), supported by ever increasing computing resources, is well developed and suitable for simulating the three-dimensional flow of large-scale structures with anisotropic turbulent scalar fluxes. Reference [8] assessed the viability and accuracy of LES for a circular cylinder at high Reynolds number, which demonstrated that the LES is more accurate than the Reynolds-Averaged Navier-Stokes (RANS). Existing work in both experiments and numerical simulations focused on the high buildings with smooth surface, while in many modern buildings the form of external elevation is diverse and grooves are more commonly used, especially in the form of balcony. In addition, the Reynolds number of most full-scale tall buildings under the condition of strong winds reach to supercritical region $\left(\mathrm{Re}>2 \times 10^{7}\right)$ for which case the wind tunnel test apparatus almost cannot meet, Hence, for large number of real-world applications, a better understanding of the effect of rectangular-groove roughness on wind characteristics at high Reynolds number under various surface conditions is required.

In the present study, both wind tunnel tests and full-scale LES are employed to investigate the effects of equidirectional-grooves with large roughness coefficients on wind characteristics of tall cylinder buildings. Firstly, models with smooth and different groove depths are tested in the wind tunnel. Then a full-scale computational domain and corresponding tall cylindrical building models were built to simulate the interaction between wind and buildings. Finally, the distribution of wind pressure coefficient, including mean pressure, and the mean drag coefficients and strouhal number dependency on Reynolds number are examined and discussed based on different groove depths.

\section{EXPERIMENTAL DETAILS}

\section{A. Wind Tunnel Tests}

The experiments were performed at wind tunnel laboratory of Zhejiang University, China. The dimensions of the working section ( $3 \mathrm{~m}$ high, $4 \mathrm{~m}$ wide and $18 \mathrm{~m}$ long) 
permitted an appropriate geometric scale and the range of available wind speeds is $3 \sim 55 \mathrm{~m} / \mathrm{s}$. Based on the cylinder diameter and the velocity, the Reynolds number ranges from $6.6 \times 10^{4}$ to $3.3 \times 10^{5}$, covering both subcritical and supercritical flows around an isolated cylinder[9]. Triangular spires and $5 \mathrm{~m}$ long fetches of floor roughness elements were employed to develop the turbulent boundary layer flows. Fig. 1 shows the experimental and theoretical profiles of mean wind speed and turbulence intensity. Also, it should be mentioned that the corresponding turbulence intensity was $\mathrm{I} z=11 \%$ approximately at the buildings height, and the wind velocity profile followed well the power law with an exponent of $\alpha=0.16$. The measured spectrum of along-wind turbulence was well agreed compared to the target Von Karman spectrum.

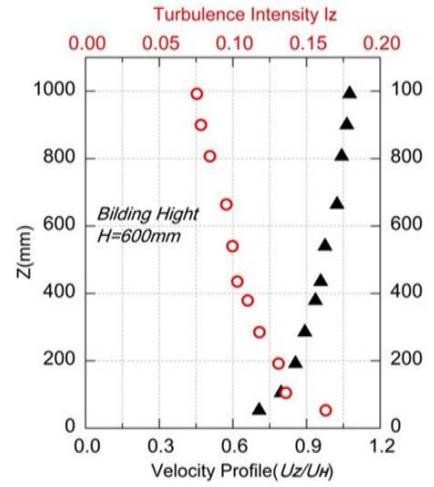

Figure 1. Wind profile imposed at model location

\section{B. Models And Measurement}

For obtaining high structural quasi-static behavior and frequencies under wind flow, much attention was paid to make the model as rigid as possible. Table 1 shows the arrangement of roughness coefficient in the wind tunnel tests and full scale sizes, respectively. All the models are made of organic glass with geometric scale of 1:300, which gives rise to the fact that the blockage ratio is $0.5 \%$. The basic model has the height of $\mathrm{H}=600 \mathrm{~mm}$ and the circular cross-section with the diameter of $\mathrm{D}=100 \mathrm{~mm}$, corresponding to $180 \mathrm{~m}$ and $30 \mathrm{~m}$ in full scale, respectively. Simultaneously, the protruding surface rough elements were made up of long-striped plastic with different roughness coefficient $t / D$ (where $\mathrm{t}$ is the size of thickness and $\mathrm{D}$ is cylinder diameter, shown in Fig. 2) and installed on the surface of the smooth models along the vertical direction, where $t / D=0$ means smooth surface.

TABLE I. SURFACE ROUGHNESS OF MODELS USED IN EXPERIMENTS.

\begin{tabular}{|c|c|c|}
\hline Models & t/D & full scale (m) \\
\hline M0 & 0 & 0 \\
\hline M1 & $1 / 40$ & 0.75 \\
\hline M2 & $1 / 20$ & 1.50 \\
\hline M3 & $3 / 40$ & 2.25 \\
\hline M4 & $1 / 10$ & 3.00 \\
\hline
\end{tabular}

In regard to the measurement, measuring points were embedded in the center of each concave. There are 20 measuring points on each level (i.e. one measuring point every 18 degrees). Each model has eight levels (marked with $\mathrm{A} \sim \mathrm{H}$ ), giving a total of 160 measuring points. Wind pressures of each tap were measured at a sampling frequency of $312.5 \mathrm{~Hz}$ and recorded simultaneously by a Pitot-static tube via a multi-channel pressure transducer connected to scanivalve and processed on a microcomputer. The total number of measured data was about 10,000 with a recording length of $32 \mathrm{~s}$ for each measuring point, corresponding to 40 min in full scale based on the time scale of $1 / 75$ and velocity scale of $1 / 4$. Due to the effects of measuring tubes, numerical compensations were employed to modify the distortions of fluctuating wind pressures by using the transfer function method[10]. Fig. 2 graphically shows the cross section of cylinder and distribution of measuring points and grooves.

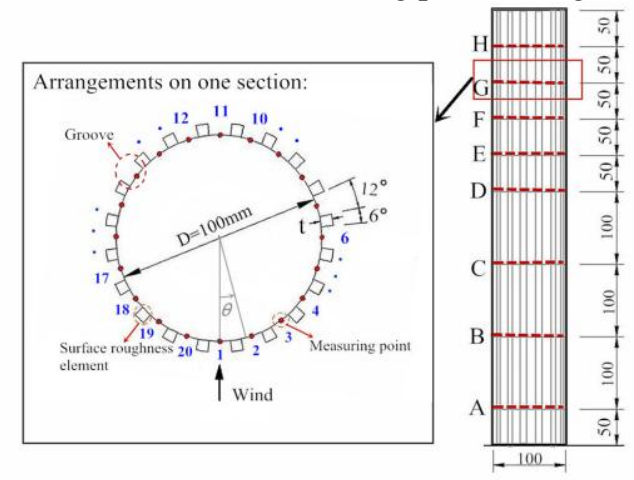

Figure 2. Arrangement of measuring points and grooves

\section{NUMERICAL SIMULATION}

\section{A. Large Eddy Simulation}

For the purpose of obtaining the wind characteristics of the cylinder comprehensively, the study carried out the three-dimensional Large Eddy Simulation based on the FLUENT module of ANSYS. In order to get rid of high frequency fluctuations, the approach of LES employed consists in filtering Navier-Stokes equations, in which the effect of small eddies is calculated using a sub-grid scale model. The filtered Navier-Stokes equations for a constant density fluid are expressed as follows:

$$
\left\{\begin{array}{l}
\frac{\partial \bar{u}_{i}}{\partial x_{i}}=0 \\
\frac{\partial}{\partial t}\left(\rho \overline{u_{i}}\right)+\frac{\partial}{\partial x_{j}}\left(\rho \bar{u}_{i} \bar{u}_{j}\right)=\frac{\partial \overline{\sigma_{i j}}}{\partial x_{j}}-\frac{\partial \bar{p}}{\partial x_{i}}-\frac{\partial \tau_{i j}}{\partial x_{j}}
\end{array}\right.
$$

where $i, j=1,2$ and $3 ; u_{1}, u_{2}$ and $u_{3}$ are the velocity components along $x, y$ and $z$ axis of the Cartesian coordinate system; $\sigma_{i j}$ is the stress tensor of filtered molecular viscosity; and $\tau_{i j}$ is the stress tensor of sub-grid scale resulting from the filtering process:

$$
\tau_{i j}=\rho \overline{u_{i} u_{j}}-\rho \overline{u_{i}} \overline{u_{j}}
$$


Among all the sub-grid models, sub-grid stress tensor is defined as Eq. (3), which is widely used in large eddy simulation.

$$
\tau_{i j}=\frac{1}{3} \tau_{k k} \delta_{i j}-2 v_{t} \overline{S_{i j}}
$$

Where, $\overline{S_{i j}}$ is the rate-of-strain tensor and $v_{t}$ is the sub-grid eddy viscosity. The isotropic part of the stresses is added to the pressure term because it has not been modeled. In the standard Smagorinsky-Lilly Model, the eddy viscosity is obtained from:

$$
v_{t}=\rho L_{S}^{2}|\bar{S}|
$$

In the equation, $|\bar{S}|=\sqrt{2 \overline{S_{i j} \overline{S_{i j}}}}$ and $L_{S}=\min \left(\kappa d, C_{S} \bar{\Delta}\right)$ are the filtered strain rate and the mixing length for sub-grid scales, respectively. $\kappa$ is the Karman constant, $d$ is the distance to the closest wall, $C_{S}$ is the Smagorinsky coefficient and $\bar{\Delta}$ is the filter width. On the basis of experience-based evaluation, the use of $C s=0.1$ provided the most accurate results $[6,11]$ and thus is also used in the present study.

\section{B. Computational Domain and Mesh}

With reference to the full-scale height $(\mathrm{H}=180 \mathrm{~m})$ and diameter $(\mathrm{D}=30 \mathrm{~m})$ of the cylinder building, a full-scale 3D computational domain was modeled with the size $55 \mathrm{D} \times 20 \mathrm{D} \times 3 \mathrm{H}(x, y, z)$. The centre of the cylinder (and the origin of the coordinate system) is placed $15 \mathrm{D}$ from the inlet boundary, $2 \mathrm{H}$ away from the top boundaries and 40D away from the outlet boundary, shown in Fig.3. A supercritical Reynolds number, ranging from $9.9 \times 10^{6}$ to $7.2 \times 10^{7}$, was facilitated by the full-scale dimensions of building and the along-wind velocity. Currently, the blockage ratio was only $1.7 \%$, which is smaller than the limitation of $3 \%$ [12].

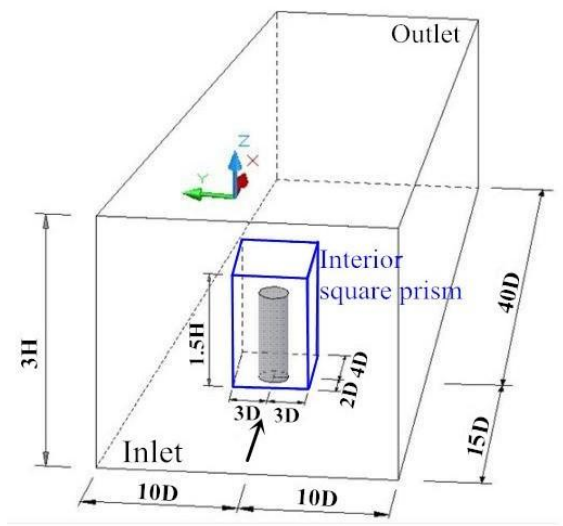

Figure 3. Computational and interior refined domain

Throughout the whole computational domain, the building was nested in an interior square prism, where the grids were refined by unstructured grid whereas structured grids distribute outside the prism. The tested building was surrounded by a viscous boundary layer with a 6-layers prism grid and the height of the first layer was small enough to satisfy the requirement of the wall unit distribution.

The mean drag coefficient $(C d)$ and the Strouhal number (St) were employed to evaluate the testing results preliminary, defined as:

$$
\begin{gathered}
C d=\frac{F_{d}}{0.5 \rho U_{r e f}^{2} D} \\
S t=\frac{n D}{U_{\text {mean }}}
\end{gathered}
$$

Where, $F_{d}$ is the aerodynamic force acting on the building in along-wind direction, $n$ is the frequency of vortex shedding, $U_{\text {mean }}$ is the mean velocity of the incoming flow and $U_{r e f}$ is the reference velocity at reference height, a point over the top of the cylinder.

\section{Simulation Assumptions And Boundary Conditions}

In order to simplify the problem, the study made the assumption that the air fluid is isotropic and incompressible and, at the same time, the fluid is Newtonian fluid, ignoring the viscous dissipation.

Numerical simulation adopted the SIMPLEC algorithm, setting boundary conditions as;

1) Inlet speed was imported using the UDF file and the inflow wind speed adopts $10 \mathrm{~m}$ height of annual average speed, namely $3 \mathrm{~m} / \mathrm{s}$, in Hangzhou area. Also, the wind speed profile function conforms to the exponential law under class-B ground roughness with an exponent of 0.16 , and -0.23 for turbulence intensity.

2) An outlet boundary condition with static pressure of 0 $\mathrm{Pa}$ was set herein, which is given relative to an operating pressure, set at atmospheric pressure. What's more, symmetrical boundary and non-slip wall condition were chose. The pressure and velocity, in present simulations, were monitored at various points within the domain. Mass conservation within the computational domain was also monitored to ensure conservation at each time step and additionally convergence criteria was set as $10^{-6}[12]$, meaning the flow field turns into a stable state.

\section{RESUlts AND Discussion}

\section{A. Validation of the Simulation}

Pressures, acquired from the tests and $L E S$, are presented as non-dimensional pressure coefficient $(\mathrm{Cp})$, defined as:

$$
C p=\frac{p-p_{0}}{\rho U_{r e f}^{2} / 2}
$$

Where, $p$ is the time-averaged pressure and $\mathrm{p} 0$ is the reference pressure, measured at a point in the upstream of the tested model. Fig. 4 presents the comparisons of mean pressure coefficient $\left(\mathrm{C}_{p \text {,mean }}\right)$ at four different degrees between numerical and experimental results, corresponding to the windward $\left(0^{\circ}\right)$, the right side $\left(90^{\circ}\right)$, leeward $\left(180^{\circ}\right)$ and the left $\operatorname{side}\left(270^{\circ}\right)$ for $\operatorname{Re}=3.3 \times 10^{5}$. While there is some 
discrepancies in quantification, it can be seen that the results of LES are agree well with experimental results, particularly in the variation tendency of $\mathrm{C}_{p \text {,mean }}$.

In addition, the discrepancies of mean drag coefficients $\left(\mathrm{C}_{d, \text { mean }}\right)$ for all the cylinders between the experimental and LES results are weeny, and the largest difference is only around $2.5 \%$. Table 2 listed the $\mathrm{C}_{d \text { mean }}$ of the cylinders acquired in LES and experiments. From what has been shown above, the data indicated that the LES are reliable.

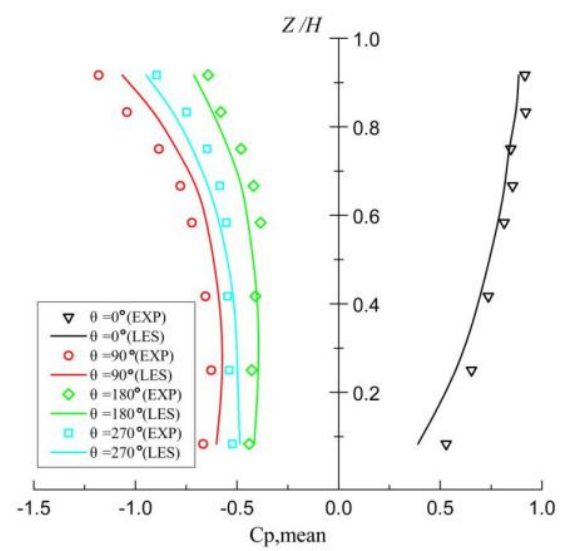

Figure 4. The comparisons of $\mathrm{Cp}$,mean for different degrees.

TABLE II. MEAN DRAG COEFFICIENTS ACQUIRED IN LES AND EXPERIMENTS.

\begin{tabular}{|c|c|c|c|}
\hline Models & LES & EXP & Difference (\%) \\
\hline M0 & 0.741 & 0.718 & 3.11 \\
\hline M1 & 1.317 & 1.290 & 2.08 \\
\hline M2 & 1.359 & 1.323 & 2.67 \\
\hline M3 & 1.370 & 1.333 & 2.73 \\
\hline M4 & 1.415 & 1.387 & 1.98 \\
\hline
\end{tabular}

\section{B. Wind Pressure Coefficient and Velocity}

Both the experiments and numerical simulations are employed to investigate the pressure on the surface of the circular cylinders since the pressure is the most direct action on the body resistance to the fluid $[1,13,14]$. Due to the presence of rough elements and the grooves, the maximum pressure occurs around $\theta=90^{\circ}$, which means that the location of the maximum pressure moves around the cylinder as a consequence of roughness elements, which disturb the structure of laminar flow glued to the cylinder surface and generate small rotating vortices in the concaves. The surface elements contribute to expanding the range of high velocity, or in other words, the roughness elements narrow the wake width due to downstream movement of the separation point, as is shown in Fig. 5. The streamline and vortex structures around the grooves are shown in Fig. 6.

\section{The Mean Drag Coefficients And Strouhal Number Dependency on Reynolds Number}

Fig. 7 show the comparisons of mean drag coefficient and Strouhal number with the published results for smooth cylinder $[3,9,15]$. Good agreement can be seen for $6.6 \times 10^{4} \leq$ $\operatorname{Re} \leq 3.3 \times 10^{5}$. Among the range of Reynolds number from 9.9 $\times 10^{6}$ to $7.2 \times 10^{7}$, however, the value of $C d$ has a trend of decrease.

Given that air flow in nature are often turbulent, which has led to the reemergence of strong, coherent shedding than that for smooth flow. Many researchers published studies on the relationship between $S t$ and $R e$. In turbulent flow of experiments, the value of $S t$ is determined by the dominant peak in the spectra of the lift fluctuations generally, corresponding to the vortices regularly shedding from the cylinder approximately. Due to the full-scale size and velocity, the test Reynolds number in turbulent flow of LES was limited in $9.9 \times 10^{6} \sim 7.2 \times 10^{7}$, and the data indicated that the $S t$ remains at the range of from 0.15 to 0.35 . For rough models, $S t$ decreased as $t / D$ and $R e$ increased. And, the $C d$ of rough cylinder is 2 3 times as large as that of smooth cylinder.
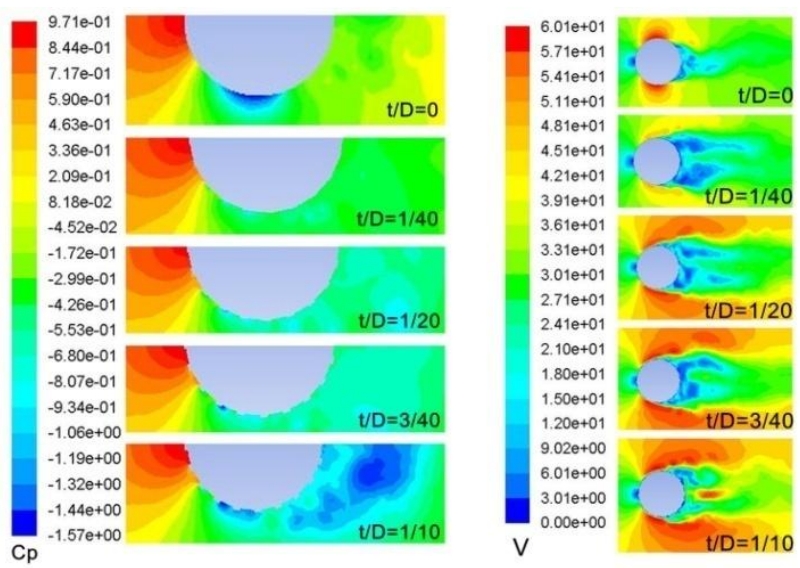

Figure 5. Contours of $\mathrm{Cp}$ and velocity magnitude.

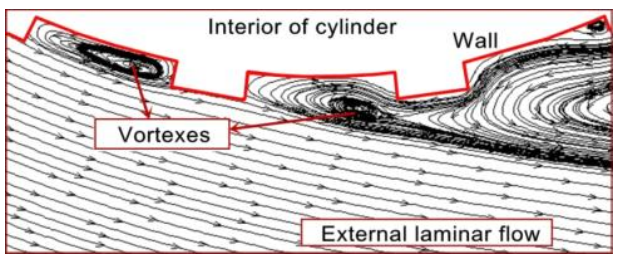

Figure 6. Details of vortexes in and around grooves.

\section{Strouhal Number Based on Various Groove Depth at $\operatorname{Re}=7.2 \times 10^{7}$}

For most full-scale tall buildings, the Reynolds number is normally greater than $2 \times 10^{7}$ under strong wind. Fig. 8 (a) shows the power spectra density $(P S D)$ of lift coefficients $\left(C_{L}\right)$ for different groove depth at $\operatorname{Re}=7.2 \times 10^{7}$. It is observed that there is always a distinct and definite peak around the frequency of 0.20 and the frequency spectrum width of across-wind $C_{L}$ narrow down due to the vortex shedding, reattached and rotating vortices.

According to the present study, surface roughness plays a role in affecting frequency in the transcritical regime and the value of peak increases with the increment of $t / D$, whereas the frequency of periodic vortex shedding has a decrease, as is reveled in Fig. 8 (b). 


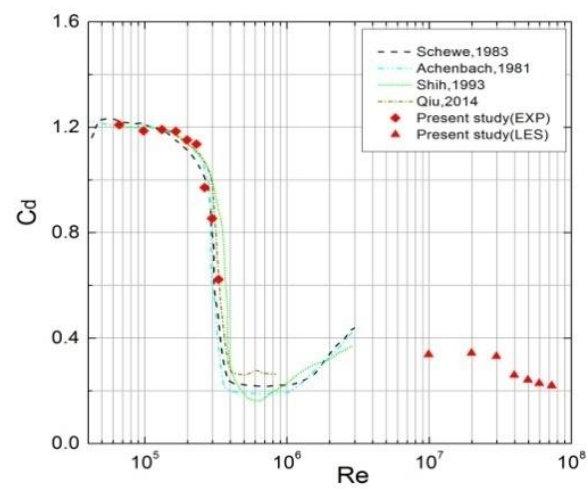

(a)

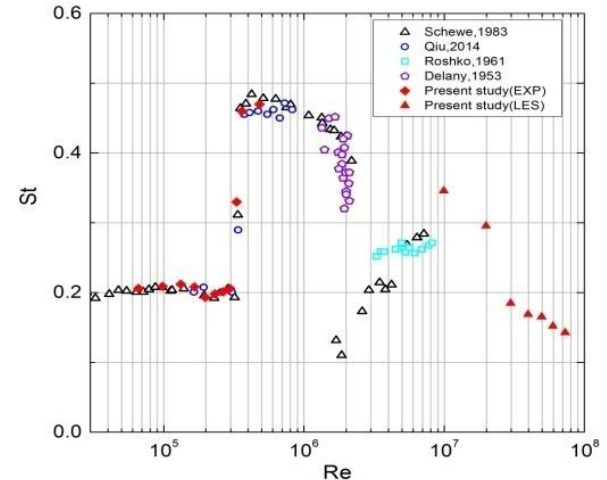

(b)

Figure 7. Comparisons of Cd and St with published research: (a) Mean drag coefficients; (b) Strouhal number

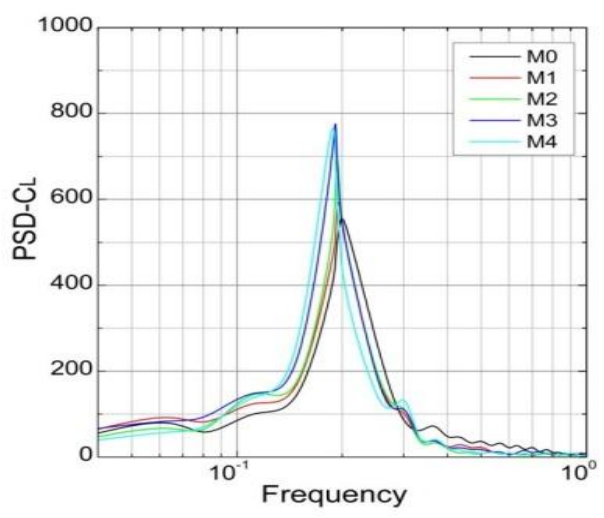

(a)

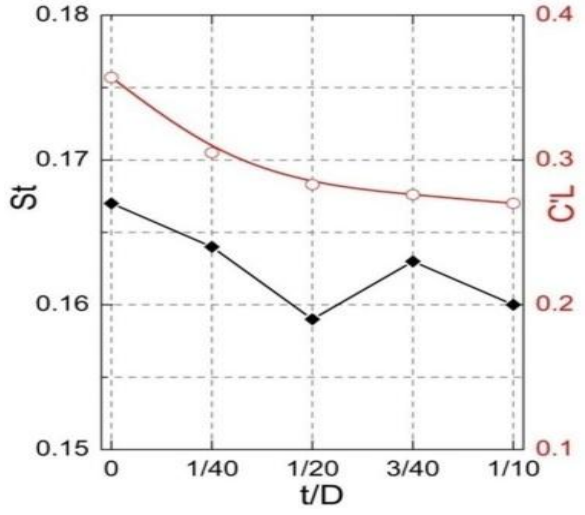

(b)

Figure 8. The wind characteristics for various roughness sizes: (a) Power spectra density; (b) St and fluctuating lift coefficients

\section{CONCLUSIONS}

From the study, using both the experiments and numerical simulations, on five cases of circular cylinders embedded with rectangular-groove roughness, the following conclusions can be drawn:

1) The convex rough elements and the rectangular grooves expand the range of high velocity and narrow the wake width due to the downstream movement of the separation point. Further, the deeper grooves cause smaller mean and fluctuating pressure. For the same level, mean pressure is highly dependent on the depth of grooves and has a reduction with the increased grooves depth.

2) For Reynolds number range from $9.9 \times 10^{6}$ to $7.2 \times 10^{7}$, $S t$ remains at the range from 0.15 to 0.35 ; and the $C d$ of rough cylinder is 2 3 times as large as that of smooth cylinder under the trend of decrease.

3) Groove roughness plays a role in affecting frequency in the supercritical regime and the frequency of vortex shedding in full-scale simulations has the value of 0.20 at $R e=7.2 \times 10^{7}$ and the frequency spectrum width of across-wind lift coefficient narrow down due to the vortex shedding, reattached and rotating vortices.

\section{ACKNOWLEDGMENT}

This research was supported by Zhejiang Provincial Natural Science Foundation of China under Grant No.LY15E080023.

\section{REFERENCES}

[1] E. Maruta, M. Kanda, and J. Sato, "Effects on surface roughness for wind pressure on glass and cladding of buildings," J. Wind Eng. Ind. Aerod. vol. 74, pp. 651-663,1998.

[2] A. Tsutomu, "Effects of surface roughness on the universal Strouhal number over the wide Reynolds number range," J. Wind Eng. Ind Aerod. vol. 69-71, pp. 399-412,1997.

[3] H. Achenbach, "On vortex shedding from smooth and rough cylinders in the range of Reynolds numbers $6 \times 10^{3}$ to $5 \times 10^{6}$ " Journal of Fluid and Mechanics 109, pp. 239-251,1981.

[4] B. Blocken, "50 years of Computational Wind Engineering: Past, present and future," J. Wind Eng. Ind. Aerod. vol.129, pp. 69-102, 2014.

[5] J. Mo, A. Choudhry, M. Arjomandi, and Y.-H. Lee, "Large eddy simulation of the wind turbine wake characteristics in the numerical wind tunnel model," J. Wind Eng. Ind. Aerod. vol.112, pp. 11-24, 2013.

[6] P. Gousseau, B. Blocken, and G. J. F. van Heijst, "Quality assessment of Large-Eddy Simulation of wind flow around a high-rise building: Validation and solution verification," Computers \& Fluids. vol.79, pp. 120-133, 2013. 
[7] K. Hanjalić, and S. Kenjereš, "Some developments in turbulence modeling for wind and environmental engineering," J. Wind Eng. Ind. Aerod. vol.96, pp. 1537-1570, 2008.

[8] P. Catalano, M. Wang, G. Iaccarino, and P. Moin, "Numerical simulation of the flow around a circular cylinder at high Reynolds numbers," International Journal of Heat and Fluid Flow. vol. 24, pp. 463-469, 2003

[9] G. Schewe, "On the force fluctuations acting on a circular cylinder in crossflow from subcritical up to transcritical Reynolds numbers," J. Fluid Mech. vol.133, pp. 265-285, 1983.

[10] Z. N. Xie, and M. Gu, "A correlation-based analysis on wind-induced interference effects between two tall buildings," Wind And Structures. vol.8, pp. 163-178, 2005.

[11] G. Hu, K. T. Tse, K. C. S. Kwok, and Y. Zhang, "Large eddy simulation of flow around an inclined finite square cylinder," J. Wind Eng. Ind. Aerod. vol.146, pp. 172-184, 2015.
[12] J. Franke, A. Hellsten, K. H. Schlunzen, and B. Carissimo, "The COST 732 Best Practice Guideline for CFD simulation of flows in the urban environment: a summary," Int. J. Environ. Pollut. vol.44, pp. 419-427, 2011.

[13] D. L. Zuo, "Full-scale measurement of wind pressure on the surface of an oscillating circular cylinders," J. Wind Eng. Ind. Aerod. vol. 133, pp. 65-79, 2014.

[14] D. A. Köse, and E. Dick, "Prediction of the pressure distribution on a cubical building with implicit LES," J. Wind Eng. Ind. Aerod. vol.98, pp. 628-649, 2010.

[15] Y. Qiu, Y. Sun, Y. Wu, and Y. Tamura, "Analyzing the fluctuating pressures acting on a circular cylinder using stochastic decomposition," Journal Of Fluids And Structures. vol.50, pp. $512-527,2014$ 